\title{
Erratum to: Physical human-robot interaction of an active pelvis orthosis: toward ergonomic assessment of wearable robots
}

Nicolò d'Elia ${ }^{1,2^{*}}$, Federica Vanetti ${ }^{2}$, Marco Cempini ${ }^{1}$, Guido Pasquini ${ }^{2}$, Andrea Parri ${ }^{1}$, Marco Rabuffetti ${ }^{3}$, Maurizio Ferrarin ${ }^{3}$, Raffaele Molino Lova ${ }^{2}$ and Nicola Vitiello ${ }^{1,2}$

\section{Erratum}

The original article [1] contained errors whereby Figure citations were incorrectly numbered and cited within the text.

The original article has now been updated to reflect the correct Figure numbers and their respective citations within the text.

\section{Author details}

'The BioRobotics Institute, Scuola Superiore Sant'Anna, viale Rinaldo Piaggio, 34, 56025 Pontedera, Pisa, Italy. ${ }^{2}$ Fondazione Don Carlo Gnocchi IRCCS, Florence, Italy. ${ }^{3}$ Fondazione Don Carlo Gnocchi IRCCS, Milan, Italy.

Received: 23 May 2017 Accepted: 23 May 2017

Published online: 05 June 2017

\section{Reference}

1. d'Elia N, et al. Physical human-robot interaction of an active pelvis orthosis: toward ergonomic assessment of wearable robots. J Neuroeng Rehabil. 2017;14:29.

* Correspondence: n.delia@sssup.it

${ }^{1}$ The BioRobotics Institute, Scuola Superiore Sant'Anna, viale Rinaldo Piaggio, 34, 56025 Pontedera, Pisa, Italy

${ }^{2}$ Fondazione Don Carlo Gnocchi IRCCS, Florence, Italy 